\title{
Analysis of DSR for Dynamic Topology Update Mechanism in LTRT based MANET's
}

\author{
Nikhil Rathod ${ }^{1}$, Sanghapal Salve ${ }^{2}$, Vikas Suryawanshi ${ }^{3}$, Yogesh $\mathrm{Wagh}^{4}$ \\ B.E (computer), Computer Department, Sinhgad Institute Of technology, Lonavala, India ${ }^{1,2,3,4}$
}

\begin{abstract}
A Wireless ad-hoc network is an autonomous, self-organize distributed, peer to peer network of fix, nomadic or mobile user that communicates over bandwidth constrained wireless links. Energy Consumption and Network Connectivity are two of the most important issue that yet to be resolve in MANET's. Broadcasting, in context of ad-hoc networks is a costly operation and thus topology control has been proposed to achieve efficient broadcasting with low interference and energy consumption. By using topology control each node optimizes its transmission power, by maintaining network connectivity by localize manner. In this paper, we propose Local Tree-Based Reliable Topology (LTRT), which is mathematically proven to guarantee k-edge connectivity while preserving the feature of Local Minimum Spanning Tree (LMST).while applying topology control technology into Manet, it is important to address the issue of performance degradation due to node mobility. In the network each node contain the topology information to maintain the connectivity with neighbors for that it needs to be frequently and appropriately update its information according to its moving speed. In our mechanism, each node determines exact value of topology control update interval according to topology information of its neighbors. By simulation results using network simulator (NS2) we achieve better dynamic topology update mechanism.
\end{abstract}

Keywords: Ad-Hoc Networks, Energy efficiency and Reliability, K-edge connectivity, LTRT,Network connectivity, PAN's, Topology control.

\section{INTRODUCTION}

1.1 Mobile Ad Hoc Network (MANET's)

A wireless Ad Hoc Network has a multi-hop relaying of packets. It can easily rapidly deployed, and expensive infrastructure can be avoided. Without an infrastructure the node handle the necessary control and networking task by themselves, generally by distributed control. Typical application are wireless PANs for emergency operation, civilian, and military use. The wireless ad hoc network is playing an increasing role in wireless networks, and wireless ad hoc networking mode has been or is being standardized in most IEEE families of wireless networks.

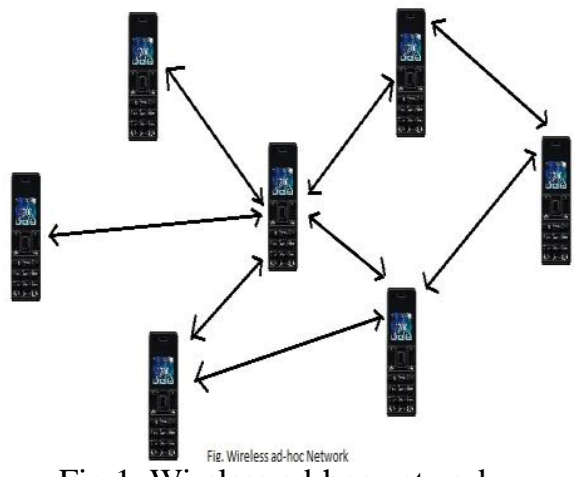

Fig.1. Wireless ad-hoc network

\subsection{Energy Efficient topology control in Manet}

In Manet, Battery power devices are used such as Smart Phones, Laptops, cellular, computers act as Nodes in Network. SavingPower Consumption while transmitting data can effectively extend the operating time of single charge. Data transmission power is proportional to square transmission range, by this power consumption can be minimized by dynamically adjusting transmission range according to distance of neighboring nodes.

\subsection{Problem definition}

The problem identified in exiting research literature is belongs to topology control in MANET is that most of the topology control algorithm do not achieve reliable and guaranteed network connectivity.

\section{RELATED WORK}

Kenji Miyao [1], Propose topology control, each node optimizes its transmission power by maintaining network connectivity in localize manner. In this paper, local minimum spanning tree [LMST] is the topology control algorithm, which has been proven to provide satisfactory performance. However, LMST always result in one connected network. In this paper local tree based reliable topology [LTRT], which is mathematically proven to guarantee while preserving the feature of local minimum spanning tree [LMST]

Atsushi Yoshinari[2], Propose produce topology update mechanism using LTRT algorithm in order to apply topology control technology into MANETs, it is essential to address the issue the performance degradation due to node mobility.

Ning $\mathrm{Li}[3]$, it presents a minimum spanning tree(MST) algorithm, called local minimum spanning tree [LMST], topology control in wireless multi-hop networks. In this algorithm each node builds its LMST independently only keeps on tree nodes that are one hop away as its neighbors in the final topology.

M.N.S. Swamy [4],it proposes the routing in Manet.Multihop communication in a WSN can be effectively overcome shadowing and path-loss effect. Routing of Wireless ad hoc networks faces several challenges, such as securing broadcast 


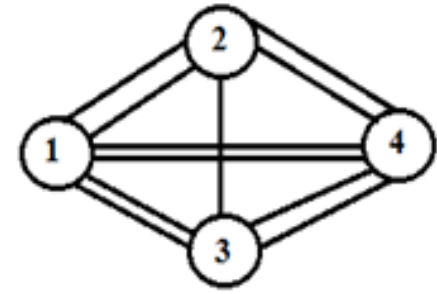

(a)

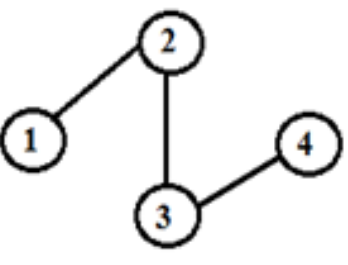

(b) LMST

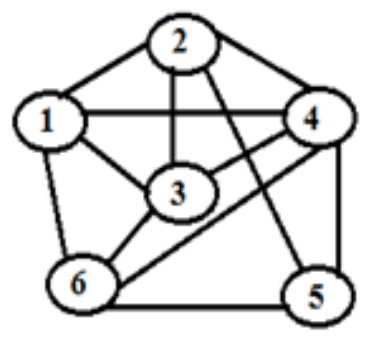

(c) $\operatorname{FLSS}(\mathrm{k}=2)$

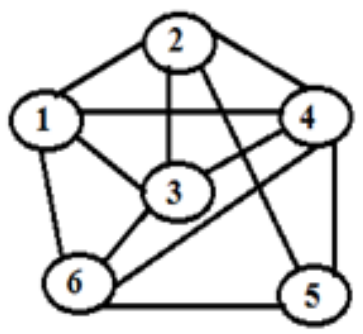

(d) $\operatorname{LTRT}(k=2)$

Fig. 2 Comparison between different topology

Communication wireless communication in an untrusted environment as well as and sending the route its self.

Rachit Jain[5], in this paper they present comparative study on the behavior of various routing protocols with path loss propagation models, various performance Metrics used for this comparison such as packet delivery fraction, average jitter, throughput and average end to end delay. It is helpful in choosing the correct protocol for any active operating environment.

The above proposed algorithms cannot be achieved realistic topology control. On other hand the proposed algorithm LTRT, preserves k-edge connectivity with much lower computational cost, $O(k(m+n \log n))$.

\section{PERFORMANCE OF DSR}

\subsection{DSR}

The DSR protocol requires each packet to carry full address, from source to the destination. This means that the protocol will not be very effective in large networks, as the amount of overhead carries in the packet will be continue to increase as the network diameter increase.

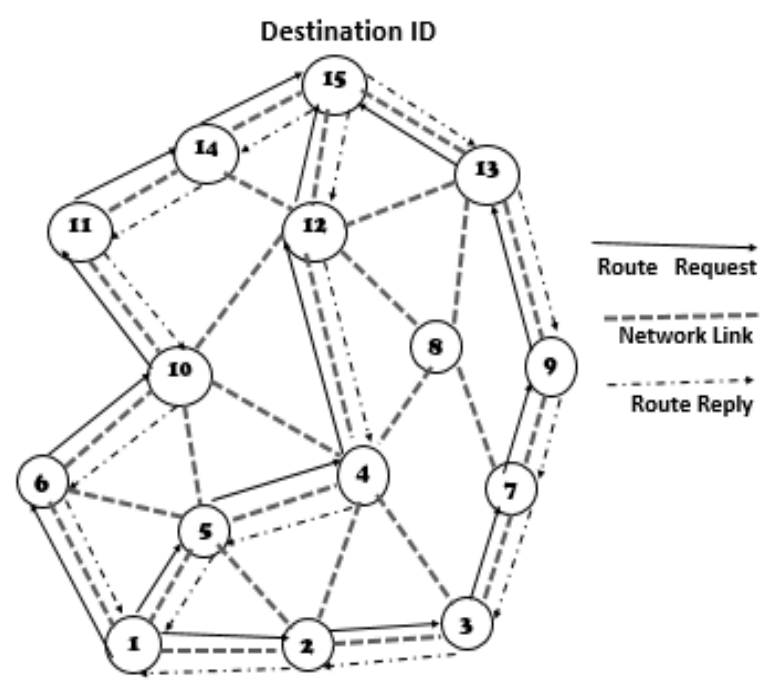

Source ID

Fig.3 Route establishment in DSR

Therefore, in highly dynamic and large networks the overhead may consume most of the bandwidth. An advantage of DSR is that nodes can store multiple routes in their route cache, which means that the source node can check its route cache for a valid route before initiating route discovery, and If a valid route is found there is no need for route discovery.

This is very beneficial in network with low mobility, because the routes stored in the route cache will be valid for a longer period of time. And it does not required any periodic beaconing, therefore nodes can enter sleep node to conserve their power.

\section{Algorithm for DSR}

\section{Define source node and Destination node}

Broadcasts a Route Request Packet (RREQ)

RREQ contains a unique Request ID and the address of the sender

If node $=$ destination node or route[destination]

Then

send a Route Reply packet (RREP)

Else if

is the source,

thendrop the packet

Else if

is already in the RREQ's route table, then drop the packet

Else append the node address in the RREQ's route table andbroadcast the updated RREQ

\section{TABLE:1 SIMUTATION SETTINGS.}

\begin{tabular}{|l|l|}
\hline Field size & $1000 \mathrm{~m} * 1000 \mathrm{~m}$ \\
\hline Maximum transmission range & $250 \mathrm{~m}$ \\
\hline Routing Protocol & DSR, AODV \\
\hline Number of nodes & 100 \\
\hline Average speed & $0-20 \mathrm{~m} / \mathrm{s}$ \\
\hline Node Placement & Random \\
\hline Traffic Type & Constant Bit Rate \\
\hline MAC Layer Protocol & IEEE 802.11 \\
\hline Propagation Model & Two Ray Ground \\
\hline Seed & 0.1 \\
\hline Simulation time & $10,000 \mathrm{~S}$ \\
\hline
\end{tabular}

\section{CONCLUSION}

The lifetime of a wireless network operating on battery power is critical to its usefulness. Network lifetime can be increased by efficiently managing the power consumption. 


\section{REFERENCES}

11] Kenji Miyao, N. Ansari and N. Kato(2009). "LTRT:An efficient and Reliable Topology Control Algorithm for Ad-hoc Network",IEEE Publications.

[2] Atsushi Yoshinari, H. Nishiyama And N. Kato, And Dan Keun Sung(2012). "Dynamic Topology Mechanism In LTRT based MANET", IEEE Publication.

[3] N. Li,J. Hou, C. Sha, and L.Sha,(2005), "Design And Analysis Of An MST-Based Topology Control Algorithm" IEEE Trans Publication.

[4] M. N. S. Swami and K. L. Du.(2010), "wireless Communication System ", Reference Book.

[5] Rachit Jain, Laxmi Shriwastava(2011),"Study And Performance Comparison Of AODV And DSR On The Basis Of Path Loss Propagation Model", International Journal Of Advance Science And Technology

[6] C. Siva Ram Murthy and B. S. Manoj (2013), "Ad-Hoc Wireless Network", Reference Book.

[7] S. Guo and O. W. W. Yang (2007), "Energy-aware multicasting in wireless ad hoc networks: a survey and discussion," Comput. Commun., vol. 30, no. 9, pp. 2129-2148,.

[8] E. L. Lloyd, R. Liu, M. V. Marathe, R. Ramanathan, and S. Ravi (2005), "Algorithmic aspects of topology control problems for ad hoc networks," Mobile Networks Applications, vol. 10, no. 1, pp. 19-34

[9] C. E. Jones, K. M. Sivalingam, P. Agrawal, and J. C. Chen(aug 2001), "A survey of energy efficient network protocols for wireless networks," Wireless Netw., vol. 7, no. 4, pp. 343-358.

[10] R. Ramanathan and R. Rosales-Hain(Mar 2000), "Topology control of multihop wireless networks using transmit power adjustment," in Proc. IEEE INFOCOM, Tel Aviv, Israel, pp. 404-413.

[11] J. Cartigny, D. Simplot, and I. Stojmenovic(Mar 2003), "Localized minimumenergy broadcasting in ad-hoc networks," in Proc. IEEE INFOCOM 2003, vol. 3, Mar. 2003, pp. 2210-2217. 